\title{
Les relations entre éducation et formation en environnement : un champ de recherche
}

\author{
Claude Croizer et Louis Goffin
}

\author{
(2) OpenEdition \\ Journals \\ Édition électronique \\ URL : https://journals.openedition.org/ere/7275 \\ DOI : $10.4000 /$ ere. 7275 \\ ISSN : 2561-2271 \\ Éditeur \\ Centr'ERE \\ Référence électronique \\ Claude Croizer et Louis Goffin, «Les relations entre éducation et formation en environnement : un \\ champ de recherche ", Éducation relative à l'environnement [En ligne], Volume 1 | 1999, mis en ligne le \\ 15 septembre 1999, consulté le 28 mai 2021. URL : http://journals.openedition.org/ere/7275 ; DOI : \\ https://doi.org/10.4000/ere.7275
}




\title{
Les relations entre éducation et formation en environnement : un champ de recherche
}

\author{
Claude Croizer et Louis Goffin
}

1 Depuis la Conférence de Tbilissi en 1977, il aura fallu un long cheminement aux chercheurs et praticiens de l'éducation relative à l'environnement (ErE) pour arriver à mettre en place des instruments conceptuels et méthodologiques à la fois satisfaisants d'un point de vue théorique et utilisable au plan pratique. On peut estimer désormais que les avancées à ce niveau sont réelles comme en a témoigné le colloque sur la recherche en ErE organisé à Montréal en novembre 1997, qui a permis de rassembler une centaine de chercheurs provenant d'une vingtaine de pays sans engendrer de querelles théoriques majeures et tout en permettant des débats approfondis. Cependant, la multiplicité des champs de recherche autour de l'ErE tend à démontrer qu'un certain nombre d'éléments conceptuels et méthodologiques reste encore à développer.

\section{La clarification des relations entre éducation et formation}

2 Le thème qui retient ici notre attention est la clarification des rapports entre les concepts d'éducation et de formation en environnement au sein des interventions destinées aux (futurs) professionnels de l'environnement. Dans un article récent (Goffin et Croizer, 1998), nous avons tenté de mettre en évidence leur complémentarité en prenant comme application les programmes d'enseignement universitaire supérieur en environnement. Nous poursuivons ici cette réflexion.

3 Les termes éducation et formation sont rarement associés et si les chercheurs en ErE ont défriché bien du terrain dans le domaine des relations entre éducation et environnement, peu de recherches ont été menées sur les questions des formations relatives à l'environnement (FrE) et sur les relations théoriques qu'entretiennent ErE et 
FrE. Nous retiendrons ici quelques axes de réflexion qui nous semblent particulièrement pertinents.

4 Sauvé (1994, p. 56) aborde cette question en opposant le caractère multidimensionnel du processus d'éducation à la notion plus restreinte (dans le temps et les objectifs) de formation : "la formation relative à l'environnement concerne la préparation à une activité professionnelle ou à des interventions particulières en matière d'environnement». La notion de formation en environnement est associée à «l'acquisition de savoirs spécifiques au sujet de l'environnement». Pour l'auteur, savoir-faire et savoir-être ne sont pour autant pas exclus du processus et il pourrait y avoir corrélation entre le fait d'être formé dans le domaine de l'environnement et la motivation à agir dans ce domaine. Des études intéressantes pourraient être menées dans cette direction, notamment pour valider l'hypothèse émise par Panneton (1994, p. 22) que la formation relative à l'environnement, en s'exerçant de manière conforme aux principes de l'andragogie, peut s'avérer pertinente pour développer, chez l'adulte, un esprit critique et une capacité d'action en faveur de l'environnement. Pour ces deux auteurs, il semble que les notions d'éducation et de formation, appliquées au domaine de l'environnement, restent proches l'une de l'autre et qu'elles soient finalement difficilement dissociables. Nous allons tenter de prolonger cette réflexion.

\section{Sur quels critères distinguer éducation et formation ?}

Le plus souvent, trois critères majeurs servent à différencier éducation et formation. Le premier critère est celui du public cible (on parle plus souvent d'éducation à propos des enfants, et de formation pour des adultes), le second critère est le temps d'application (on éduque à l'école et on forme à l'Université, en entreprise), le troisième est relatif à la durée du processus (la formation serait ponctuelle et l'éducation continue).

Nous proposons de retenir un autre critère de différenciation, celui de leur finalité.

7 Par « éducation ", nous faisons référence à un processus global et continu centré sur le devenir de la personne (Goffin et Croizer, 1998). Dans ce sens, les savoirs et savoir-faire transmis au cours du processus éducatif ne sont que des moyens qui ont comme finalité le développement de savoir-être et de savoir-agir transférables dans les actes de la vie quotidienne.

8 L'activité de « formation » (prise ici en tant que formation spécialisée dans le secteur de l'environnement) met l'accent sur le transfert de connaissances et de compétences dans un but professionnel. On s'adresse à la personne en tant que membre d'une communauté socioéconomique. L'acquisition de savoirs et de savoir-faire est la finalité essentielle, tant pour les organisateurs de formation que pour les participants. Pour autant, l'acquisition de ces compétences ne se justifie que par leur caractère opérationnel, leur mise en œuvre dans le cadre d'activités appliquées au domaine de l'environnement. La mobilisation de ces compétences traduit un savoir aigre environnemental.

9 Si l'on s'en tenait à ces définitions, les relations entre éducation et formation en environnement pourraient alors être claires et relativement simples car elles se caractériseraient par une différence d'accent, de degré : l'éducation relative à l'environnement concernerait en priorité les sphères individuelles et sociales; la formation relative à l'environnement serait centrée sur la sphère professionnelle. 
10 Éducation et formation auraient donc toutes deux comme finalité le «savoir-agir » en tant que capacité à transposer en actes les compétences intellectuelles et techniques que l'on peut mobiliser ainsi que les valeurs dont on est porteur. Ce savoir aigre se traduirait soit dans les gestes de la vie privée et sociale, soit dans ceux de la vie professionnelle. Pour autant, cette distinction nous semble simpliste et insatisfaisante. Il nous semble en effet qu'en matière d'environnement particulièrement (mais cela est vrai pour d'autres types désignés d'éducation), les sphères individuelles et professionnelles sont en étroite interaction.

11 Le processus qui mène à l'intervention environnementale procède à la fois de l'éducation (par le développement d'une éthique personnelle) et de la formation (par la préparation à l'exercice de responsabilités professionnelles). Le critère de finalité constitue donc un des éléments à considérer, mais reste insuffisant pour rendre compte à lui seul de la complexité des rapports entre les notions d'éducation et de formation.

12 Comme il semble difficile et peu pertinent de poursuivre une réflexion sur les liens entre éducation et formation en environnement sans aborder une problématique concrète, nous proposons d'étudier le cas des formations supérieures en environnement.

\section{Les formations supérieures en environnement}

13 Nos recherches sur l'évaluation des formations postgraduées en environnement nous amènent à poser la question de l'adéquation entre les formations proposées aux futurs professionnels de l'environnement, les tâches qu'ils auront à remplir et les spécificités de l'intervention en environnement. En clair, les formations proposées répondent-elles à la fois aux objectifs des organisateurs, aux attentes des participants et à celles, peutêtre différentes, de leurs futurs employeurs et enfin aux exigences d'une société qui se déclare concernée par la mise en œuvre d'un développement "durable»? Cette question renvoie aussi au débat contemporain sur les rôles et missions de l'université qui, en plus de sa mission éducative traditionnelle (promouvoir l'esprit critique, la créativité, l'autonomie de recherche), doit faire face à des exigences académiques accrues - la recherche de l'excellence - et à des pressions fortes de ses clientèles (étudiants, employeurs, pouvoirs publics) pour se recentrer vers des objectifs de préparation à l'emploi.

14 Il semble par ailleurs reconnu par les instances internationales (à Tbilissi en 1977, à Rio en 1992 et à Thessalonique en 1998) que les problèmes d'environnement que nous connaissons n'ont pas comme unique origine des insuffisances technologiques ou des causes naturelles, mais sont aussi en partie liés à nos modes de consommation, à nos comportements individuels, etc. Si nous acceptons cette vision des faits, nous posons alors la question du mandat des institutions proposant des formations qualifiées en environnement. S'agit-il uniquement pour celles-ci d'apporter des connaissances et compétences scientifiques, techniques, méthodologiques ou bien ces formations en environnement doivent-elles également intégrer les dimensions éthiques et sociales de l'environnement, c'est-à-dire apporter des capacités de réflexion permettant de clarifier les valeurs sous-jacentes à tout choix de stratégie environnementale? Observons, à partir de deux exemples, comment sont présentés les objectifs de formations supérieures en environnement. 


\section{Des objectifs annoncés et des objectifs implicites}

15 Notre réflexion s'appuiera sur l'étude des programmes de deux formations postgraduées en sciences de l'environnement, l'une en Europe, l'autre en Amérique du Nord.

\section{Exemple 1 : La Fondation Universitaire Luxembourgeoise (Belgique)}

Les objectifs généraux des enseignements (notamment le programme de DES en sciences de l'environnement) sont de permettre aux étudiants :

- D'acquérir une formation générale pluridisciplinaire en sciences de l'environnement en complément de leur formation universitaire spécialisée de départ ;

- D'acquérir des connaissances spécialisées dans certains domaines des sciences de l'environnement ;

- D'acquérir des méthodes de gestion de l'environnement ;

- De développer une capacité de travail en équipe pluridisciplinaire ;

- D'appliquer les méthodes acquises à la gestion de problèmes environnementaux, etc.

\section{Exemple 2 : Université du Québec à Montréal, Institut des sciences de l'environnement}

La formation vise à permettre aux étudiants de :

- Comprendre et évaluer l'articulation entre la dynamique des systèmes naturels et la dynamique du développement économique et social ;

- Développer des compétences permettant de participer, par la mise en commun d'expertises disciplinaires, thématiques ou sectorielles, à la définition de la gestion et la résolution de problèmes environnementaux dans le but de contribuer à la mise en œuvre du développement durable.

18 Ces objectifs représentent les cahiers des charges que se fixent ces institutions. Il est révélateur que l'on écrive dans un cas « enseignement » et dans l'autre «formation » à propos de programmes comparables. La politique en matière d'enseignement supérieur au Québec est en effet résolument orientée vers une mission de formation, la Communauté française de Belgique est de son côté en plein débat sur le devenir de son université. Dans la pratique quotidienne, c'est cependant aussi le terme de formation qui est le plus largement utilisé par les professeurs et les étudiants de la Fondation Universitaire Luxembourgeoise (FUL).

L'examen de nombreux descriptifs de formations postgraduées en environnement révèle une communauté apparente dans les projets de formation proposés aux étudiants. Bien que l'inter et la multidisciplinarité soient au centre des propos et que l'on se propose de réconcilier «sciences de la terre » et "sciences de l'homme», la formulation de ces objectifs reste ancrée dans une représentation positiviste de l'appréhension des questions environnementales. Les verbes opérateurs utilisés (acquérir, développer, appliquer, comprendre, etc.) laissent peu de place à l'incertitude dans laquelle baignent pourtant les choix de stratégies et politiques environnementales.

Tels que formulés, ces objectifs ne reflètent cependant qu'imparfaitement les réalités que ces formations impliquent. En filigrane derrière les objectifs généraux apparaissent 
d'autres objectifs tels que la formation à l'esprit critique, à l'autonomie, à la prise en compte de la complexité et de la globalité dans l'analyse de problématiques environnementales. Ces formations sont effectivement (et c'est une dimension importante) des lieux de débats sur des controverses environnementales ou valeurs et représentations sociales s'affrontent. Chaque enseignant, quelle que soit sa discipline, laisse transparaitre sa vision du monde, ses choix personnels, ses propositions de solutions et contribue ainsi au débat environnemental. Une enquête auprès d'anciens étudiants (Croizer et von Frenckell, 1998) montre d'ailleurs que ces aspects ont été pour eux une dimension importante de leur formation.

Évidemment, l'atteinte de tels objectifs apparaît difficile à évaluer par les organisateurs de formation, car ce sont sans doute les participants eux-mêmes qui sont le mieux placés pour juger, par exemple, du développement de leur esprit critique ou de leur autonomie de réflexion et de recherche.

Ces objectifs «implicites » contribuent selon nous pleinement à une réelle formation des futurs professionnels de l'environnement et participent à l'affirmation d'une déontologie environnementale.

En ce sens, être capable, par exemple, de réfléchir aux interconnexions entre les disciplines et aux divergences d'opinions fait partie intégrante du savoir-faire des professionnels de l'environnement. Ces aspects de la formation restent difficiles à traduire en réalités pédagogiques. Former à l'interdisciplinarité, par exemple, suppose davantage que la simple tolérance des autres disciplines; elle nécessite une réelle capacité à travailler avec l'autre, ce qui peut, au moins en partie, s'apprendre en formation.

L'analyse des programmes de formation mentionnés ci-dessus (et d'autres exemples vont dans le même sens) révèle une forme de convergence entre organisateurs, participants, pouvoirs publics et employeurs sur ce qui est attendu des professionnels de l'environnement. La formation à un ensemble de connaissances scientifiques et de compétences techniques est proposée de manière explicite. Les aspects dialectiques, le développement personnel des participants et la clarification des systèmes de valeurs sous-jacents à toute décision environnementale restent implicites.

\section{Quelques pistes de recherche}

En premier lieu, il pourrait être pertinent d'étudier, en situation pédagogique, l'alternance entre le rôle de formateur et celui d'éducateur joué par l'enseignant. À quel moment un enseignant cesse-t-il d'être formateur pour devenir éducateur ? Quels sont les indices qui pourraient nous permettre de dire que telle partie de l'activité relève d'une démarche de formation et que telle autre s'inscrit dans une perspective éducative? Une telle recherche pourrait faire apparaître, dans les interventions pour les professionnels de l'environnement, des critères propres à l'acte de formation et d'autres critères indiquant un contexte d'éducation. À titre d'hypothèse, on pourrait avancer que des activités comme la transmission de connaissances scientifiques, l'étude et la mise en application de méthodologies, les études de cas, les visites de terrain et les travaux pratiques, etc. s'apparentent à une démarche de formation et que les temps de débats sur l'épistémologie de l'environnement, les controverses, la part laissée à la 
réflexion individuelle relèvent d'une démarche éducative. Il est bien entendu qu'au sein des formations que nous connaissons, les deux types d'activités sont présents, s'imbriquent étroitement et que la définition de ces critères nécessiterait une recherche en profondeur. Dans la pratique, les formations supérieures en environnement nous semblent bien s'inscrire dans une perspective éducative sans que celle-ci soit nécessairement mise en évidence.

Cela nous amène à un deuxième axe de recherche qui concernerait les dimensions implicites et explicites des objectifs de formation en environnement. Notre expérience d'étudiant, puis de formateur en sciences de l'environnement nous amène à dire que ce qui tient du processus éducatif en situation de formation reste le plus souvent dans l'implicite. Se pose alors la question de savoir si les pratiques de formation en environnement gagneraient à expliciter des objectifs et des modes de fonctionnement qui relèvent peut-être d'une sorte d'alchimie entre l'enseignant et son groupe d'étudiants. Nous pouvons aussi émettre l'hypothèse que sont naturellement laissés dans l'ombre les aspects des formations qui se révèlent les plus délicats à évaluer. Mais est-il nécessaire, et possible, de vouloir tout évaluer?

\section{BIBLIOGRAPHIE}

Croizer, C. et von Frenckell, M. 1998. L'évaluation des formations universitaires postgraduées en environnement : le cas de la Fondation Universitaire Luxembourgeoise, rapport de recherche. Arlon, Belgique : Fondation Universitaire Luxembourgeoise.

Fondation Universitaire Luxembourgeoise. (1997). Programme d'enseignement, 1997-1998. Arlon, Belgique : Fondation Universitaire Luxembourgeoise.

Goffin, L. et Croizer, C. (1998). Éducation et formation en environnement : deux perspectives complémentaires. European Environmental Management Review, 11, 20-23.

Institut des sciences de l'environnement. (1997). Tiré à part du programme de maîtrise en sciences de l'environnement. Montréal, Québec : Université du Québec à Montréal.

Panneton, F. (1994). Formation relative à l'environnement : design d'un module de formation en gestion environnementale intégrée à l'intention des décideurs de PME. Mémoire de maîtrise en sciences de l'environnement, Université du Québec à Montréal.

Sauvé, L. (1994). Pour une éducation relative à l'environnement. Montréal et Paris : Guérin et Eska.

\section{AUTEURS}

\section{CLAUDE CROIZER}

Claude Croizer est titulaire d'un master en gestion de l'environnement et d'une maîtrise en sociolinguistique. Il est actuellement assistant de recherche à la Fondation Universitaire Luxembourgeoise et prépare son doctorat sur l'évaluation des formations supérieures en 
environnement. Son expérience professionnelle a trait au domaine de l'éducation des adultes, notamment dans les pays en développement.

\section{LOUIS GOFFIN}

Louis Goffin est licencié agrégé en philosophie et lettres, docteur en sciences de l'environnement, aspects sociologiques, chercheur en pédagogie de l'environnement, professeur à la Fondation Universitaire Luxembourgeoise et directeur de cette institution depuis 1995. Il est également président fondateur en Belgique du Réseau IDEE, ONG pour l'Information et la Diffusion en Éducation relative à l'Environnement. 